\section{PSICOLOGÍA IBEROAMERICANA}

\section{Psicología lberoamericana}

ISSN: 1405-0943

psicología.iberoamericana@uia.mx

Universidad Iberoamericana, Ciudad de

México

México

Hernández Jiménez, Natalia

Reflexiones sobre Marcas en la Piel

Psicología Iberoamericana, vol. 18, núm. 1, enero-junio, 2010, pp. 38-46

Universidad Iberoamericana, Ciudad de México

Distrito Federal, México

Disponible en: http://www.redalyc.org/articulo.oa?id=133915936005

- Cómo citar el artículo

- Número completo

- Más información del artículo

Página de la revista en redalyc.org

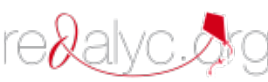

Sistema de Información Científica

Red de Revistas Científicas de América Latina, el Caribe, España y Portugal

Proyecto académico sin fines de lucro, desarrollado bajo la iniciativa de acceso abierto 


\title{
Reflexiones sobre Marcas en la Piel
}

\author{
Reflections About Skin Marks
}

Natalia Hernández Jiménez

Facultad de Psicología Universidad Intercontinental, CD. de México*

\section{RESUMEN}

En el siguiente trabajo se desarrolla un análisis de contenido de las transcripciones realizadas en los talleres de cine debate y grupo de reflexión realizados en el Centro de Tratamiento para Varones durante el 2007. Dichos proyectos fueron llevados a cabo por la Facultad de Psicología de la Universidad Intercontinental. Se encontró que aproximadamente 20 reclusos, de edades entre los 17 y 21 años, marcan su piel voluntariamente con cicatrices, existiendo variantes en las significaciones. Aunado al análisis se plantea un recorrido teórico psicoanalítico el cual aborda el tema de las marcas en la piel vinculando dichos actos con el pasaje al acto y el acting out, al igual que el papel que tiene el dolor en la realización de las marcas. Los resultados de la investigación se vinculan con la imposibilidad que tienen los jóvenes para verbalizar sus estados internos.

Descriptores: Adolescentes, cicatrices, dolor, pasaje al acto, acting out.

\section{ABSTRACT}

The following project develops a content analysis of the conversation transcriptions from the Cinema Debate and Reflection Groups workshops conducted by the Psychology Faculty of the Universidad Intercontinental, which took place in 2007 at the Centro de Tratamiento para Varones. The main result of the workshop evidenced that approximately 20 inmates, aged between 17 and 21, voluntarily scar themselves, and that each scar bears a different meaning. Alongside the content analysis, a theoretical and psychoanalytical approach is followed. This approach touches upon scarring, linking it to phenomena such as "pasaje al acto" and acting out, as well as the role pain plays in the scarring process. The results of the investigation are related to the subjects' impossibility to verbalize their internal emotional states.

Key words: Adolescents, scars, pain, enactment, acting out.

\section{INTRODUCCIÓN}

A través del tiempo, las marcas en la piel, las cicatrices y los tatuajes, han tenido diferentes significados y formas de ser interpretados, tanto por quienes lo realizan, como por aquellos que los observan. Las reflexiones aquí descritas acerca del tema están relacionadas con mi práctica dentro del Centro de Tratamiento para Varones durante el 2007, a través de la cual tuve oportunidad de abordar distintos temas gracias a los grupos de cine debate y de reflexión. La investigación consistió en el análisis de cada una de las transcripciones realizadas en todas las sesiones de cine debate y grupo de reflexión que abordaban el tema de las marcas en la piel y después fue enriquecida por medio de lecturas y del análisis de fuentes especializadas en el tema que fundamentaran los posibles motivos de realización de las marcas, al igual que las variantes en significaciones.

\footnotetext{
* Natalia Hernández Jiménez. Facultad de Psicología de la Universidad Intercontinental. Valle 109 Jardines del Pedregal, Álvaro Obregón, C.P. 01900. México D.F. Teléfonos: 55684071 cel: 0445527290394 email: nataliahj@gmail.com.
} 
Dentro de la misma investigación nació la idea del rol que juega la piel en la formación de marcas, así como la mirada de un 'otro' dentro de la simbolización de estados internos para los reclusos, y de qué manera tales marcas pueden facilitar la expresión psíquica.

A partir de la revisión de libros, capítulos de libros, artículos de revistas y, en gran parte, de estudios realizados dentro del Centro de Tratamiento para Varones, así como de investigaciones vinculadas a las marcas de la piel y de las diversas finalidades de las mismas, se creó una recopilación importante de datos con miras a lograr una aproximación de los motivos y de las significaciones que tenían las marcas en la piel para los jóvenes. Por lo tanto, la pregunta central de la investigación sería: ¿Qué significado tienen las marcas en la piel para los jóvenes internos en el Centro de Tratamiento para Varones?

Distintos autores e investigadores como Doctors (2007), Reisfeld (2004) y Nasio (2007), han formulado hipótesis acerca de las razones por las cuales las personas rasgan de manera voluntaria su piel sin una finalidad suicida y además, de qué es lo representan estas marcas para ellos y para quienes los observan. Durante muchos años, las marcas en la piel han sido objeto de estudio para médicos y psicólogos, pero con el paso del tiempo y los cambios socioculturales relacionados, estas se han convertido en uno de lo temas más intrigantes para el psicoanálisis.

A pesar de que las marcas han existido desde tiempo remotos, la función de las mismas ha ido variando, tal como lo menciona Reisfeld (2004) dentro de su obra Tatuajes, una mirada psicoanalítica:

En efecto, sus funciones fueron múltiples: se utilizaron como señal de realeza; símbolo de devoción religiosa; para marcar la transición del joven a la adultez; como distintivo de clan o tribu; como un medio de identificación personal o una forma de mostrar valor o virilidad; como estímulo de atracción sexual; como talismán para alejar a los malos espíritus; como parte necesaria de los ritos funerarios; para diferenciar a la mujer casada de la casadera; como muestra de amor; como forma de marcar o identificar esclavos, marginados y convictos (p. 22).
Por su parte, Doctors (2007) en el artículo Avances en la comprensión y tratamiento de la autolesión en la adolescencia ahonda en los motivos por los cuales los adolescentes se autolesionan la piel:

La reacción de quien se corta pertenece en gran parte a una experiencia de inminente catástrofe psicológica que, hablando subjetivamente, es "sentida" como amenazante. Una necesidad urgente de gestionar una experiencia desorganizadora de vulnerabilidad está en el centro del episodio de autolesión. Desesperadas circunstancias psicológicas exigen medidas extremas. Lo que es más difícil de entender del síntoma es lo siguiente: la conducta considerada problemática por los observadores (padres, profesores, amigos, y terapeutas), es vivida por el adolescente como una manera de solucionar problemas.

Finalmente esta formación y demostración de las marcas en la piel de los reclusos se vincula con lo que García (2003) describe en el artículo Cuando Eros tienta a Tanatos como tipo de acto sintomático en relación al acting out:

Un acto sintomático tiene un carácter metafórico y tiene forma discursiva aunque no sea verbal. Las acciones impulsivas las conceptualizamos como puesta en acto o "acting out". En el "acting out" (to act out), término que incluye tanto la idea de representación teatral como la de actuar, está siempre la dirección a otro y una cierta intencionalidad de afectación a otro, en busca de respuesta o quizás de desciframiento. Es inconsciente tanto el saber de su actuación como los sentidos o intencionalidades que vehiculizan o producen y su aparición parece hablar de algo que salió de circuito simbólico pero apelando o dirigiéndose a otro (p. 87).

A lo largo del presente artículo, se hace un recorrido teórico además de un apartado de abstracciones del análisis de las transcripciones de los grupos de cine debate y grupo de reflexión con el objetivo de encontrar aproximaciones en relación a las finalidades y significaciones que tienen las marcas en la piel para los jóvenes reclusos del Centro de Tratamiento para Varones, aunado a la idea de vincular dichas marcas con el acting 
out y el pasaje al acto a pesar de que ambas definiciones pueden ser similares.

\section{METODOLOGÍA}

Los grupos de cine debate y de reflexión son una derivación de grupos focales en donde se pretendía que los jóvenes reclusos abordaran los temas centrales de la investigación (violencia, sexualidad, familia y abuso de sustancias), permitiendo ahondar en otros temas como las marcas en la piel. Estaban conformados por alrededor de 20 jóvenes de entre 17 y 21 años de edad los cuales fueron elegidos por el reclusorio de manera aleatoria como parte de sus actividades extracurriculares, considerando por lo tanto a la investigación con un tipo de muestreo aleatorio simple. La duración de ambos grupos fue de un año (enero a diciembre de 2007). Las sesiones de trabajo en el Centro de Tratamiento para Varones no partían de preguntas previamente estructuradas ni de una metodología relacionada a entrevistas abiertas, ya que el medio de obtención de información tenía su origen en la proyección de películas o simplemente de una discusión abierta con duración de una hora que permitía la expresión libre de los jóvenes para hablar de cualquier tema. En cuanto a las limitaciones de la investigación, al hacer las transcripciones globales de las sesiones de trabajo, se generalizaba al grupo, lo cual limitaba el conocimiento exacto de la cantidad de jóvenes que marcaban su piel. Las transcripciones se analizaron de manera general y grupal, no particularmente.

Con el fin de identificar el lugar y el significado de las marcas en la piel, se analizó una sesión representativa del total de las sesiones llevadas a cabo, además de una revisión teórica de diferentes aproximaciones psicoanalíticas que describen y explican por qué los jóvenes marcan su piel de manera voluntaria.

\section{Transcripciones}

Extracto de la sesión de grupo de cine debate de la película "Hoolingans"

Patio 3, Sección 1

Fecha: 18 de abril de 2007

\section{${ }_{-}$El pertenecer a un grupo es importante?}

- No tenemos de otra, aquí finalmente te sirve, la banda no te toma por aislado.

-Sí, por que te está comiendo la cárcel.
- ¿Qué es eso de que te coma la cárcel?

-A veces estamos en depresión y la misma cárcel te come.

- Todos necesitamos de todos.

-Cuando estamos mal platicamos entre nosotros, jugamos, nos charrasqueamos y así nos alivianamos y se va el tiempo más fácil.

- ¿Qué es charrasquear?

-Esto, las cortadas que nos hacemos.

-Así se les dice.

-Son cortadas que nos vamos haciendo.

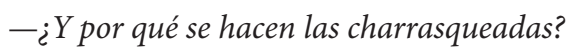

-Porque se siente bien.

-Se te olvida todo cuando te las haces.

- Se ven chingones.

$-{ }_{i}$ No les duele?

-A veces, pero se ve chido.

-¿Qué les duele más?

-A veces duele, pero aliviana.

-¿Qué aliviana?

-Lo que se tiene en la cabeza, nuestras preocupaciones.

- ¿Cómo se sienten de estar aquí?

-Estamos hartos.

- De la chingada.

- Me he dado cuenta de que les es muy difícil estar aquí...

-Sin tele.

-Sin actividades.

-Lo que me llama la atención es ¿ustedes saben qué repercusiones tenía el hacer ciertas cosas?

-Debemos de tomar los riesgos y encontrar beneficios.

- ¿Y qué pueden hacer entonces si saben los riesgos?

-Decidimos correr el riesgo.

-Allá afuera no lo ves tanto, te sientes superman pero adentro es cuando te das cuenta. 
- De las personas que están por segunda vez ¿qué piensan de conocer y continuar? ¿Les gusta estar aquí? - Lo que hicimos fue un error.

-Es el ambiente en el que vivimos.

-Es que sales pensando que vas a cambiar, pero el mismo ambiente te influencia y vuelves a lo mismo porque no te pasa nada.

- ¿Y hay algún punto en el que te pongas a pensar antes de hacerlo?

- Sabes por que ya lo viviste y sí piensas en las consecuencias.

-Son tus propias decisiones.

—Quieres la vida fácil y mejor.

- ¿Esto es entonces para darse y dar una mejor vida? -Quieres darte una vida mejor y a tu chava también.

— Se olvidan de ti, rara es la que se aguante.

- Se me hace raro que en diferentes sesiones han mencionado que es su decisión las cosas que hacen y ahora menciona que es el ambiente ¿qué factor importa más? -Pues es que al que le gusta, le gusta y al que no, no. -Depende...

-Es que no sabemos lo que queremos y cuando quieres algo pues le echas ganas.

— ¿Cómo les haces para saber qué quieres?

-Experimentando.

-Convicción de lo que quieres.

- No nos damos la oportunidad de ver distintos caminos.

-Si quisiéramos hacerlo, lo haríamos. Es muy fácil que te digan lo que hagas pero no te dicen cómo hacerlo por que no es cotidiano.

- ¿Es entonces equiparable o valió la pena la satisfacción del momento?

-No, hubo momentos chidos pero no vale la pena.

- Las horas se hacen eternas.

-A la familia la vemos poco.

- Valoramos a la familia y a las mujeres.

— ¿Servirá hablar de estas cosas entre ustedes?

- Sí, te desahogas.
- Cuando ves y escuchas te da cuerda para que te caiga el veinte.

-Además hablando entre nosotros te das cuenta que las familias son iguales, familias disfuncionales.

- ¿Qué es una familia disfuncional?

-Con problemas, madre alcohólica.

-Violencia entre los hijos y a la esposa.

- ¿Eso influencia?

-Sí, nuestras conductas, pero no el que estemos aquí.

- No tuvimos reconocimiento en casa.

- ¿Lo hablan con la familia?

-No, da pena.

- ¿Les importa lo que pensemos o pensáramos de ustedes?

-Ni idea.

-Nos hemos formado con el qué dirán.

-Qué tal que piensen lo peor de nosotros por estar aquí.

-Nosotros nos ponemos las etiquetas de que somos unos culeros.

- ¿Los veríamos igual afuera?

- No, pero aquí ya nos ven por el delito.

-A lo mejor y no.

-Por las etiquetas que nos ponemos.

- ¿Ese mismo acto o delito es para subir de rango o les da mala imagen?

-No lo hacemos por eso, buscamos el reconocimiento de nuestra sociedad, búsqueda de identidad.

\section{- ¿Les es importante pertenecer a un grupo?}

- Como no lo tuvimos en casa lo buscamos en otra parte.

-Quieres pertenecer o embonar, esa es nuestra búsqueda.

- ¿Pero si mencionaron que no embonaban antes por qué la extrañan?

-Porque la perdimos y la valoramos ahorita. 
- ¿Tienes que perder las cosas para valorarlas?

-Depende...

-Es una forma de pensar.

- Mejor darte cuenta antes.

\section{Recorrido teórico}

\section{El significado de la piel y las marcas en ella}

El que se tome la piel como uno de los ejes centrales de la investigación, se debe a que esta tiene distintas significaciones desde el estudio psicoanalítico así como una función peculiar para la realización de las marcas de los jóvenes del Centro de Tratamiento para Varones. Para ahondar sobre el tema, se recurre a varios estudios que aportan aproximaciones sobre las posibles significaciones de la piel para los jóvenes reclusos.

Distintos psicoanalistas han realizado investigaciones en las que se han dedicado de lleno a estudiar el significado que tiene la piel desde un plano físico, psíquico y la manera en la que se representan los estados internos y mentales de los seres humanos.

La piel puede ser considerada como la protección de los seres humanos hacia el exterior y más específicamente como la vestimenta con la que se relaciona con el mundo. También se le ha relacionado con la forma en la que se involucra y vincula con el amor de la madre.

Silvia Reisfeld en la obra Tatuajes, una mirada psicoanalítica, propone que

La piel que recubre el cuerpo opera como un primer límite entre el organismo y el mundo externo. Al mismo tiempo, recibe estímulos que provienen tanto del interior como del exterior. Es a partir de esta doble faz que cumple múltiples funciones para el desarrollo del aparato psíquico (2004, p. 46).

En Tres ensayos sobre una teoría sexual, Sigmund Freud (1905), afirma que la piel debe ser considerada como la zona erógena por excelencia, ya que en ella pueden tornarse un cúmulo de excitaciones placenteras. La piel reviste particular erogeneidad en el caso de los impulsos de crueldad (sadismo) y dolor (masoquismo), ambos constitutivos de la pulsión sexual.

Debido a que la piel sufre de diferentes variaciones y modificaciones a lo largo de la vida, los cambios en la apariencia externa no sólo repercuten en la represent- ación del esquema corporal, sino que también reflejan la forma en la que el individuo se ve a sí mismo y en la manera en que se muestra a los demás.

La piel marcada de los reclusos refleja, según Garma (1970), los sentimientos pasados en la relación con su madre:

Originariamente el tatuaje pudo intentar reproducir el aspecto de la piel del recién nacido, teñida por la sangre materna y el meconio, así como su arrugamiento provocado por el paso a través del estrecho conducto genital materno [... ] la finalidad primaria del tatuaje, como la de los vestidos, sería conservar el amor y la protección materna (p. 204).

Finalmente, Lidia Agazzi (2006) en el artículo Escrituras en el cuerpo menciona lo siguiente en relación a las marcas en la piel:

A mi parecer se trata de una práctica mediante la cual se elabora un producto que, tentativamente, llamaré cuerpo-dolor. Cabalgando entre el pasaje al acto y la restitución delirante, el cuerpo-dolor no alcanza a ser síntoma, no retorna desde lo reprimido, no es una formación de compromiso efecto del inconsciente, sino que escribe en el mapa de la piel un borde cicatrical, una marca, un punto focal narcisizado, que le permite al sujeto recuperar la sensación de estar vivo, liberándolo de las representaciones angustiosas que estaban a punto de hacerlo enloquecer (p. 82).

\section{El Dolor}

Para los jóvenes del Centro de Tratamiento para Varones, el dolor es aspecto que puede variar en cuanto a objetivos y finalidades. Para aproximarse al tema y con la finalidad de descifrar el significado que los jóvenes reclusos le atribuyen a este, encontramos aproximaciones psicoanalíticas que ahondan en ello y que a lo largo del proyecto se integrarán con la discusión del análisis de las transcripciones.

$\mathrm{Al}$ igual que en el caso de la piel, el significado del dolor en psicoanálisis abarca muchas variantes, una de ellas es la aportación que hace Juan David Nasio (2007) al mencionar que: 
Desde el punto de vista psicoanalítico no existe diferencia entre el dolor físico y el dolor psíquico o, más exactamente, entre la emoción dolorosa provocada por una sensación dolorosa y el dolor psíquico propiamente dicho. Ello se debe, a que el dolor es un fenómeno mixto que surge del límite que se establece entre el cuerpo y la psique (p. 15).

Por su parte, Freud considera al dolor como una seudopulsión, la cual se justifica por ser así, pues “intenta ligar las excitaciones tras una efracción intensa, que atraviesa los umbrales defensivos (protección al estímulo)" (Agazzi, 2006, p. 83). Además lo define de la siguiente manera: "Pero el dolor, esta seudopulsión, tiene el único objetivo de detener la alteración del órgano y el displacer que la acompaña [...]. Además, el dolor es imperativo; sólo obedece a la acción del tóxico que lo supone" (Freud, 1905, p. 43).

Juan David Nasio (2007) hace un análisis acerca de la imagen de la herida, del cual podríamos apoyarnos para entender por qué los jóvenes reclusos se marcan la piel con elementos cortopunzantes:

Esta imagen mental de la herida, nacida de la percepción de la lesión, fija el dolor vivido en un lugar preciso del cuerpo. Al sentir dolor, la persona quemada cree que su dolor se concentra en la herida y sólo emana de ésta, es decir, de la abertura del tejido, como si la fuente del sentimiento se redujera la extensión de la quemadura (Nasio, 2007, p. 19).

Por otro lado, Katia Weissberg (2007) en el artículo Los caminos del dolor menciona que:

Un órgano lastimado produce una corriente de energía interna, devastadora y no dominada, que sumerge al yo en un estado de invasión; la homeostasis del sistema psíquico ha quedado rota y el principio del placer que la regula ha sido momentáneamente abolido. Es entonces cuando el yo, aunque perturbado, consigue autopercibir su propia turbación. Esta singular percepción por parte del yo de su estado de conmoción interna se traduce como emoción dolorosa, a la vez que ubica dentro del cuerpo la fuente interna de la que parte. Este flujo masivo de excitación tiene dos consecuencias: la primera es la inscripción de una imagen anémica, en algunas de esas neuronas, del evento; la segunda se refiere a una excitabilidad acrecentada del conjunto neuronal (p. 22 ).

Las aproximaciones descritas anteriormente nos adentran en la idea de que, para los jóvenes el Centro de Tratamiento para Varones, el dolor funciona como un mecanismo que les permite manipular sensaciones corporales al igual que procesos psíquicos. El dolor físico para ellos tendrá una función general y particular en relación con la formación de marcas en la piel.

\section{Del Pasaje al Acto}

Hablar del acting out y del pasaje al acto podría ser algo complicado y complejo, ya que ambos se considerarían similares en cuanto a que se vinculan con el actuar.

El Diccionario de Psicoanálisis de Laplanche (1967) define el término "actuar" de la siguiente manera:

El término Agieren, como también el de "actuar", implica un equívoco, que es el propio pensamiento de Freud: éste confunde lo que en la transferencia, es actualización con el hecho de recurrir a la acción motriz, la cual no se halla necesariamente implicado por la transferencia (Laplanche 1967, p. 10).

El "pasaje al acto" (en inglés enactment) es diferente al "acting out". Sin embargo, no están tan alejados uno del otro, ya que ambos son expresión de una forma de repetición. Se podría considerar como una primera diferencia la característica de ser consciente de lo que motiva el acto. Para Lander (2007):

El pasaje al acto refiere al acto conciente que se realiza después de una lucha con la angustia: expresión del conflicto del pasado infantil, entre el deseo y la prohibición. Se refiere a un acto realizado por un sujeto con capacidad de pasar a la acción, después de vencer la inhibición y la angustia.

De igual manera Lander (2007) describe cómo dentro del Seminario 10, Lacan (1963a) menciona que el pasaje al acto se trata de un acto como sujeto; esto finalmente establece el hecho de que el acto envuelve inevitablemente al otro: 
Me refiero al deseo del otro. El pasaje al acto, es un acto en el que el analista se encuentra comprometido ya que ha producido una respuesta propia "en acto". Igual sucede con "el pasaje al acto" realizado fuera del encuadre analítico. Allí existe un otro a quien va dirigida la acción. Si el otro no existe, no responde, o no se involucra, entonces no existe el pasaje al acto. Pasa a ser un acto como síntoma (una contra-fobia o un acto obsesivo) o quizás un acting out.

Esto último va a depender de la claridad de consciencia de la motivación al acto. Finalmente, Lacan (1963) refiere que en el pasaje al acto "el sujeto se queda en la escena". Por lo que al involucrar al otro en el acto, quien a su vez responde con otro acto, la situación se transforma en un asunto entre dos y así se mantiene un vínculo entre ambos.

\section{DISCUSIÓN}

A partir de la revisión teórica y del análisis de las transcripciones realizadas en el Centro de Tratamiento para Varones, se podría mencionar que las marcas en la piel se vinculan con dos factores determinantes: El primero de ellos nos remite a que, con la finalidad de evitar el dolor psíquico, los jóvenes marcan su piel por medio del dolor físico. Como segundo factor, el dejar marcas en la piel se relaciona con el significado que tiene el acontecimiento de ingresar y vivir dentro de dicha institución.

La manera en que los jóvenes se refieren a su familia y amigos refleja la posición entre el rechazo y la integración en que su entorno social los coloca, ya que a pesar de que sienten rechazo de sus familiares y de la sociedad por los delitos que cometieron, las marcas que se realizan los integran al ámbito delictivo proporcionándose a sí mismos la imagen con la que serán reconocidos entre ellos cuando se encuentren fuera de la institución. Si partimos de las definiciones teóricas, encontramos que para los jóvenes del Centro de Tratamiento para Varones, la piel es un lienzo en donde las cicatrices, marcas y rastros que se realizan de forma voluntaria, permiten que los sentimientos de rechazo, violencia y desagrado cobren vida exterior e interiormente. Esto se vincula con un método de valoración y apropiación del cuerpo, ya que así se otorgan a sí mismos el poder de controlar las sensaciones placenteras y las desagradables.
Las marcas son un medio de comunicación entre los jóvenes y su cuerpo, y entre ellos y los que los observan, ya que éstas los distinguirán en el rol de reclusos al salir de la institución; funcionarán como recordatorios constantes de los delitos por el que ingresaron al Centro de Tratamiento para Varones. ¿Qué es el joven infractor con una marca en la piel dentro y fuera de la institución? La respuesta de dicha pregunta nos remite a la etimología la palabra stigma, traducida en los modernos diccionarios como una marca que implica haber caído en estado de desgracia o desaprobación. Para los jóvenes tal desaprobación o desgracia no es del todo desagradable, ya que recurren voluntariamente a las marcas por la necesidad de reconocerse, así como también en rechazo de todo contacto con la autoridad. La mirada de los reclusos a sus marcas en la piel durante el ingreso y salida de la institución, nos remiten al pensamiento de Jean Paul Sartre (1943) citado por Silvia Reisfeld (2004) en Tatuajes, una mirada psicoanalítica: "Con la mirada ajena, la situación se me escapa (...) yo ya no soy dueño de la situación” (Sartre, 1943). Finalmente la misma autora concluye mencionando que " la expansión actual del tatuaje nos habla de un determinismo de la mirada (...) esto es, una tendencia activa a procurar ser mirado y reconocido como entidad subjetiva” (Reisfeld, 2004, p. 62).

Los jóvenes del Centro de Tratamiento para Varones dan la idea de que una parte fundamental de haberse realizado marcas y cicatrices en la piel, tenía como finalidad la de "alivianar" el dolor psíquico por medio del dolor físico. El dolor físico para los jóvenes tendría el significado de una nula elaboración lingüística de todos aquellos procesos internos por los que están atravesando. A pesar de que los jóvenes reclusos sientan dolor al realizarse las marcas, el poder hacerlas cuando ellos lo desean, habla de lo importante que es para ellos tener control absoluto de sus propias sensaciones corporales. El dolor psíquico es devaluado y negado por el dolor físico al realizarse las marcas, como si por medio de la visualización de estas, los sentimientos desaparecieran gracias a que el dolor se concentra únicamente en la herida que se han generado. La problemática y la significación de encontrarse en la situación de reclusión ha quedado fijada en la piel y no en la psique. Esto podría hacer pensar que las marcas son una especie de pasaje al acto, como Lacan lo menciona, ya que a diferencia 
con el acting out en donde se genera un mensaje simbólico para el Otro, el pasaje al acto es una huída respecto del Otro hacia la dimensión de lo real; el pasaje al acto representa una salida de la red simbólica, una disolución del lazo social aunque no necesariamente implica una psicosis subyacente, entraña de todos modos una disolución del sujeto. Por un momento el sujeto se convierte en puro objeto.

El vinculo que se encuentra entre las marcas y el pasaje al acto se debe a que los jóvenes del Centro de Tratamiento para Varones realizan estas marcas con la finalidad de que sean observadas, así los jóvenes vinculan a un "otro" y otros en la realización de las mismas. El solo hecho de que las marcas le proporcionen a los jóvenes una mejor manera de compartir una experiencia que evita el dolor psíquico, hace de igual manera pensar en estas como un acting out.

En las marcas en la piel se encuentra implícita la relación que existe entre los jóvenes de la misma institución; entre ellos con sus padres: con la autoridad; con el resto de psicólogos con los que trabajan; y con los que realizaron la investigación durante las sesiones de cine debate como de grupo de reflexión. Las marcas de los jóvenes son respuestas a los sentimientos de reclusión y de depresión manifiestos, en donde involucran el entorno en el que se desenvuelven.

El objetivo de la realización de las marcas en la piel es una fusión entre la imposibilidad de los jóvenes por verbalizar sus sentimientos y el acto de dejar marcado un pasaporte que les permitirá ingresar de nuevo a la

\section{REFERENCIAS}

Agazzi, L. (2006). Escrituras en el cuerpo, sus afecciones. Reflexiones Psicoanalíticas. México: Círculo Psicoanalítico Mexicano

Doctors, S. (2007). Avances en la comprensión y el tratamiento de la autolesión en la adolescencia. Aperturas psicoanalíticas. Revista Internacional de Psicoanálisis, 27. Recuperado el 12 de julio de 2009 en http://www. aperturas.org/articulos.php?id=0000060\&a=Avances-en-la-comprension-y-tratamiento-de-la-autolesion-en-la-adolescencia situación delictiva y a la vida marginal fuera del reclusorio.

La pregunta que queda en el aire por lo tanto es: ¿se deberá evitar que los jóvenes maquen su piel? Como se mencionó anteriormente, la piel y las marcas son medios de expresión y del lenguaje no verbal que permiten a los jóvenes manifestar sus estados internos. Sin embargo, el hecho de que estas sean actos autodestructivos hacia su fisionomía nos remite a que los jóvenes actualmente no han encontrado las herramientas suficientes para verbalizar motivos latentes ante su situación y que existe una constante desvalorización hacía sí mismos. Evitar las marcas en la piel finalmente generará que la sintomatología autodestructiva se manifieste por medio de alguna otra conducta, un ejemplo sería el abuso de sustancias o la violencia física.

No se debe de evitar que los jóvenes expresen libremente su sentir, tal como se planteaba en el objetivo de la investigación con los grupos de cine debate y de reflexión. El énfasis y el objetivo con los reclusos del Centro de Tratamiento para Varones debe consistir en modificar la manera en la que recurran a la expresión, lo cual provocará que la actuación de los sentimientos se transforme en palabra y por lo tanto, esto replanteará la autopercepción que tienen de sí mismos. Seguir recurriendo a la actuación y no a la verbalización funciona en los jóvenes como reforzador de la idea de transgresión a las normas, a su cuerpo y a la autoridad.

Freud, S. (1905). Tres ensayos sobre teoría sexual. En Obras Completas. Vol. 2. Madrid: Biblioteca Nueva.

García, J. (2003). Cuando Eros tienta a Tanathos. Revista Uruguaya de Psicoanálisis. Vol. 27. 74-94p.

Garma, Á. (1970). Nuevas aportaciones al psicoanálisis en los sueños. Argentina: Paidós.

Lacan, J. (1963a). Pasaje al acto y acting out. Seminario IX. Argentina: Paidós.

Lacan, J. (1963b): La angustia. Seminario X. Inédito. Argentina: Paidós. 
Lander, R. (2007). Lógica del acting out y el pasaje al acto. Recuperado el 04 de enero de 2010 en http://www. elsigma.com/site/detalle.asp? IdContenido=11421

Laplanche, J. \& Pontalis, J. B. (1967). Diccionario de psicoanálisis. Argentina: Paidós.

Nasio, J. D. ( 2007). El dolor físico. Argentina: Gedisa.
Reisfeld, S. (2004). Tatuajes: una mirada psicoanalítica. Argentina: Paidós.

Sartre, J. P. (1943). El ser y la nada. Argentina: Losada. Weissberg, K. (2007). Los caminos del dolor. Reflexiones Psicoanalíticas. México: Círculo Psicoanalítico Mexico.

Fecha de recepción: enero 2010

Fecha de publicación: mayo 2010 